\title{
Zinc supplement greatly improves the condition of parkin mutant Drosophila
}

\author{
Saini, N ; Schaffner, W
}

\begin{abstract}
Parkinson's disease (PD) is a progressive neurodegenerative disorder in which oxidative stress is implicated as a major causative factor. Mutations in the gene encoding Parkin, a ubiquitin ligase, are responsible for a familial form of PD. In a Drosophila disease model lacking Parkin (park(25) null mutant), we tested the effect of zinc supplementation. Zinc is an essential trace metal and a component of many enzymes and transcriptional regulators. Unlike copper and iron, zinc is not redox-active and under most conditions serves as an antioxidant. We find that the condition of parkin mutants raised on zinc-supplemented food is greatly improved. At zinc concentrations where controls begin to show adverse effects as a result of the metal supplement, parkin mutants perform best, as manifested in a higher frequency of reaching adulthood, extended lifespan and improved motoric abilities.
\end{abstract}

DOI: https://doi.org/10.1515/bc.2010.052

Posted at the Zurich Open Repository and Archive, University of Zurich

ZORA URL: https://doi.org/10.5167/uzh-36739

Journal Article

Published Version

Originally published at:

Saini, N; Schaffner, W (2010). Zinc supplement greatly improves the condition of parkin mutant Drosophila. Biological Chemistry, 391(5):513-518.

DOI: https://doi.org/10.1515/bc.2010.052 


\section{Short Communication}

\section{Zinc supplement greatly improves the condition of parkin mutant Drosophila}

\author{
Nidhi Saini and Walter Schaffner* \\ Institute of Molecular Life Sciences, University of Zürich, \\ Winterthurerstrasse 190, CH-8051 Zürich, Switzerland \\ * Corresponding author \\ e-mail: walter.schaffner@imls.uzh.ch
}

\begin{abstract}
Parkinson's disease (PD) is a progressive neurodegenerative disorder in which oxidative stress is implicated as a major causative factor. Mutations in the gene encoding Parkin, a ubiquitin ligase, are responsible for a familial form of PD. In a Drosophila disease model lacking Parkin (park ${ }^{25}$ null mutant), we tested the effect of zinc supplementation. Zinc is an essential trace metal and a component of many enzymes and transcriptional regulators. Unlike copper and iron, zinc is not redox-active and under most conditions serves as an antioxidant. We find that the condition of parkin mutants raised on zinc-supplemented food is greatly improved. At zinc concentrations where controls begin to show adverse effects as a result of the metal supplement, parkin mutants perform best, as manifested in a higher frequency of reaching adulthood, extended lifespan and improved motoric abilities.
\end{abstract}

Keywords: antioxidant; metal homeostasis; metallothioneins; MTF-1; Parkinson's disease; zinc transporters.

Parkinson's disease (PD), characterized by the loss of dopaminergic (DA) neurons in the substantia nigra, is a progressive neurodegenerative disorder with the second highest incidence rate and is the most common age-related movement disorder (Olanow and Tatton, 1999; Dawson and Dawson, 2003; Greene et al., 2003). Both genetic and environmental factors contribute to its pathogenesis. Oxidative stress is considered to be a major factor in the pathogenesis of PD, as evidenced by an elevated content of redox-active iron and lipid peroxides in the diseased brain, impaired mitochondrial function, and alterations in the antioxidant defense mechanisms (Dexter et al., 1989; Jenner and Olanow, 1996; Greene et al., 2003; Pesah et al., 2004). Mutations in six genes, including parkin which encodes an E3 ubiquitin ligase, have been associated with rare, early-onset, familial forms of PD (West and Maidment, 2004; Gasser, 2005; Sang et al., 2007). Interestingly, some alleles of these genes might be susceptibility factors for environmental toxins (Choi et al., 2000; Warner and Schapira, 2003; Bueler, 2009).

In our study, we used a Drosophila melanogaster line in which the ortholog of the human parkin gene is disrupted by transposition of a P-element (Greene et al., 2003; Pesah et al., 2004). Parkin mutant flies present with male and female sterility (Riparbelli and Callaini, 2007), mitochondrial and muscle abnormalities, locomotor defects, an inability to fly owing to degeneration of indirect flight muscles, increased sensitivity to multiple stresses, including oxidative stress, and a severely reduced lifespan (Palacino et al., 2004; Greene et al., 2005; Whitworth et al., 2005). Some of these defects arise because parkin mutants have dysfunctional mitochondria with disturbances in the electron transport chain. In mice, in contrast to its pivotal role in humans (Choi et al., 2000), Parkin function does not seem to be critical for the survival of DA neurons (Goldberg et al., 2003; Itier et al., 2003; Palacino et al., 2004). Similarly, Drosophila that are null mutants for parkin do not generally display DA neuron loss (Greene et al., 2003; Pesah et al., 2004), although a partial loss of DA neurons in the PPL1 cluster of the brain has been reported (Whitworth et al., 2005).

In the late 1980s, the antioxidant function of the redoxinert metal zinc was recognized and proposed to be mediated by the protection of protein sulfhydryl groups and/or by competing against redox-active metals (Bray and Bettger, 1990). Additionally, zinc ions can upregulate the expression of metallothioneins, which owing to their high cysteine content can serve as antioxidants. Zinc has an established antiapoptotic function that minimizes ROS-induced cellular oxidative damage (Suzuki et al., 1991). This also occurs in the central nervous system, particularly in the brain (Kocaturk et al., 1996).

More than 70 different enzymes involved in the metabolism of biomolecules require zinc as a cofactor (Parkin, 2004). Zinc is an integral part of the hundreds of transcription factors that contain zinc finger domains (Berg and Shi, 1996), and it plays a role in cellular signal transduction and in modulation of synaptic neurotransmission. Zinc is critical for the growth and regulation of cells and alterations in zinc metabolism have been implicated in causing neurological dysfunctions on the one hand, and on the other hand providing neuroprotection. Maintenance of intracellular zinc homeostasis is thus an essential requirement in all living organisms (Valko et al., 2005).

The best characterized zinc-activated transcription factor is the metal response element-binding transcription factor-1, 


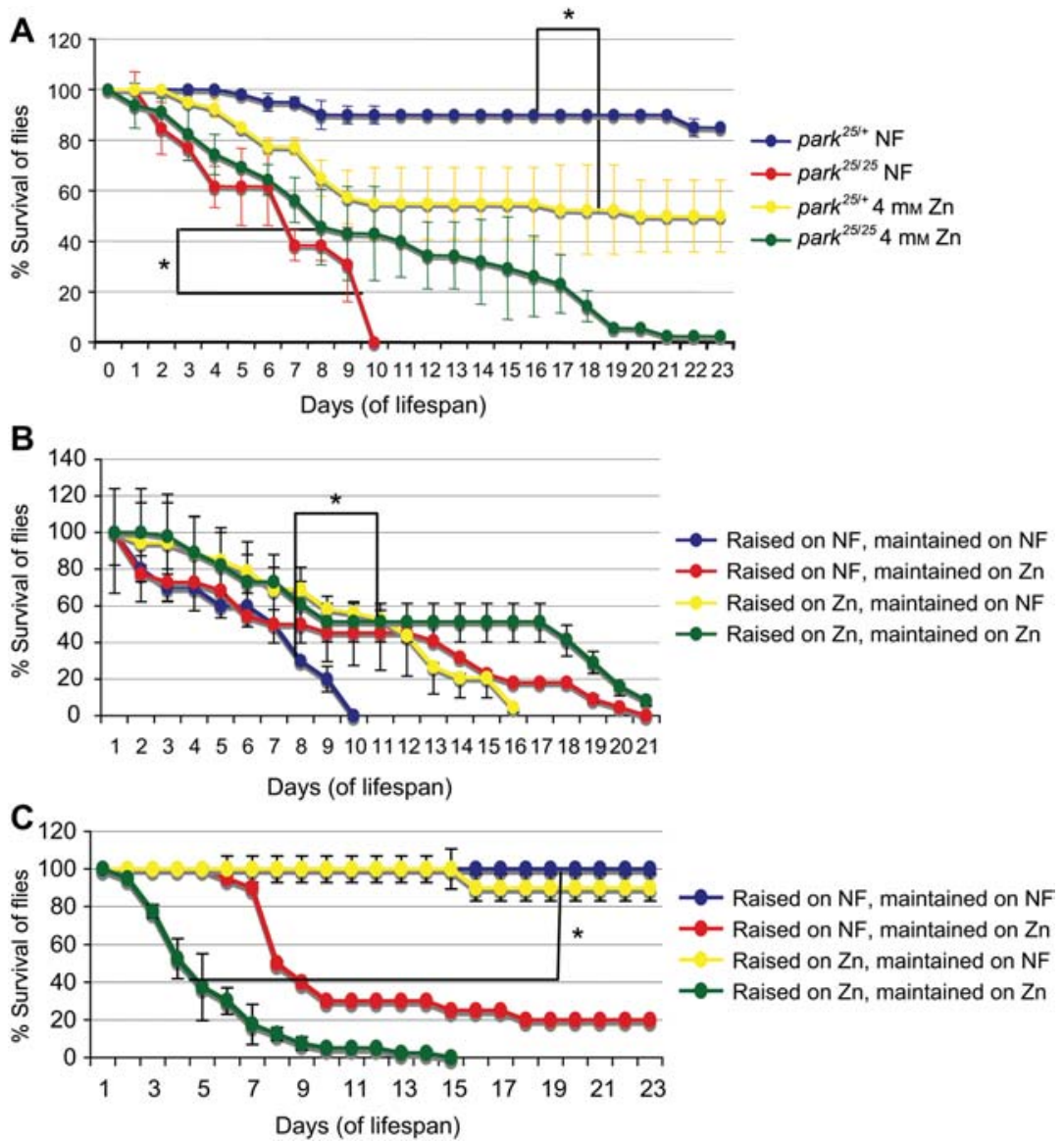

Figure 1 Drosophila parkin mutants show an enhanced lifespan on zinc-supplemented food.

(A) Zinc supplementation ( $4 \mathrm{~mm} \mathrm{ZnCl}_{2}$ ) increased the longevity of the parkin mutant flies (park ${ }^{25 / 25}$ ). The maximum lifespan of 11 days on normal food (NF) increased to 23 days. Control flies ( park $^{25 /+}$ ) showed an opposite effect with a reduction of lifespan on zinc food. (B) Survival of park $^{25 / 25}$ flies and (C) of park ${ }^{25 /+}$ control flies raised and maintained on NF or zinc-supplemented food. Error bars indicate standard deviation. The significance between survival curves was analyzed using the Kaplan-Meier log-rank statistical test $(* p<0.01)$. Methods: for survival assays, NF was supplemented with zinc chloride to a final concentration of 4 mM. One- to two-day-old flies (20 per vial) were maintained at $25^{\circ} \mathrm{C}$ on a $12: 12 \mathrm{~h}$ light/dark cycle for each genotype in triplicate vials. Surviving flies were transferred to fresh food vials every 2 days and counted daily. As a control, flies of the same genotype were grown on food without metal supplement. In each lifespan assay testing different conditions, the controls of $\operatorname{park}^{25 /+}$ and park $^{25 / 25}$ flies raised on NF were the same. The variations in the median lifespan of control flies in different experiments can be attributed to subtle experimental variations.

also referred to as metal-responsive transcription factor-1 (MTF-1). In response to zinc, MTF-1 translocates from the cytoplasm to the nucleus where it regulates the expression of several genes, notably metallothioneins and some zinc transporters (Andrews, 2000; Smirnova et al., 2000; Lichtlen and Schaffner, 2001; Saydam et al., 2001). MTF-1 requires elevated zinc concentration for DNA binding. This property was exploited for the activation of MTF-1-dependent pro-

Table 1 Zinc increases the frequency of parkin mutant Drosophila reaching adulthood.

\begin{tabular}{|c|c|c|c|}
\hline Food condition & Genotype & park $^{25 / 25} /$ total progeny & $\%$ of park $^{25 / 25}$ \\
\hline \multirow[t]{2}{*}{ Normal food } & Set 1 park $^{25 / 25}$ & $31 / 1116$ & $2.8 \%$ \\
\hline & Set 2 park $^{25 / 25}$ & $10 / 450$ & $2.2 \%$ \\
\hline \multirow[t]{2}{*}{$4 \mathrm{~mm} \mathrm{Zn}$} & Set 1 park $^{25 / 25}$ & $110 / 611$ & $18 \%$ \\
\hline & Set 2 park $^{25 / 25}$ & $186 / 956$ & $19 \%$ \\
\hline
\end{tabular}

For the analysis of eclosure frequency, egg laying was allowed for 6 days and the parent population (10 males and 10 females each of park $^{25 /+}$ ) was the same in all vials of normal food (NF) or zinc ( $\mathrm{Zn})$, and progeny flies were counted at the same time. 

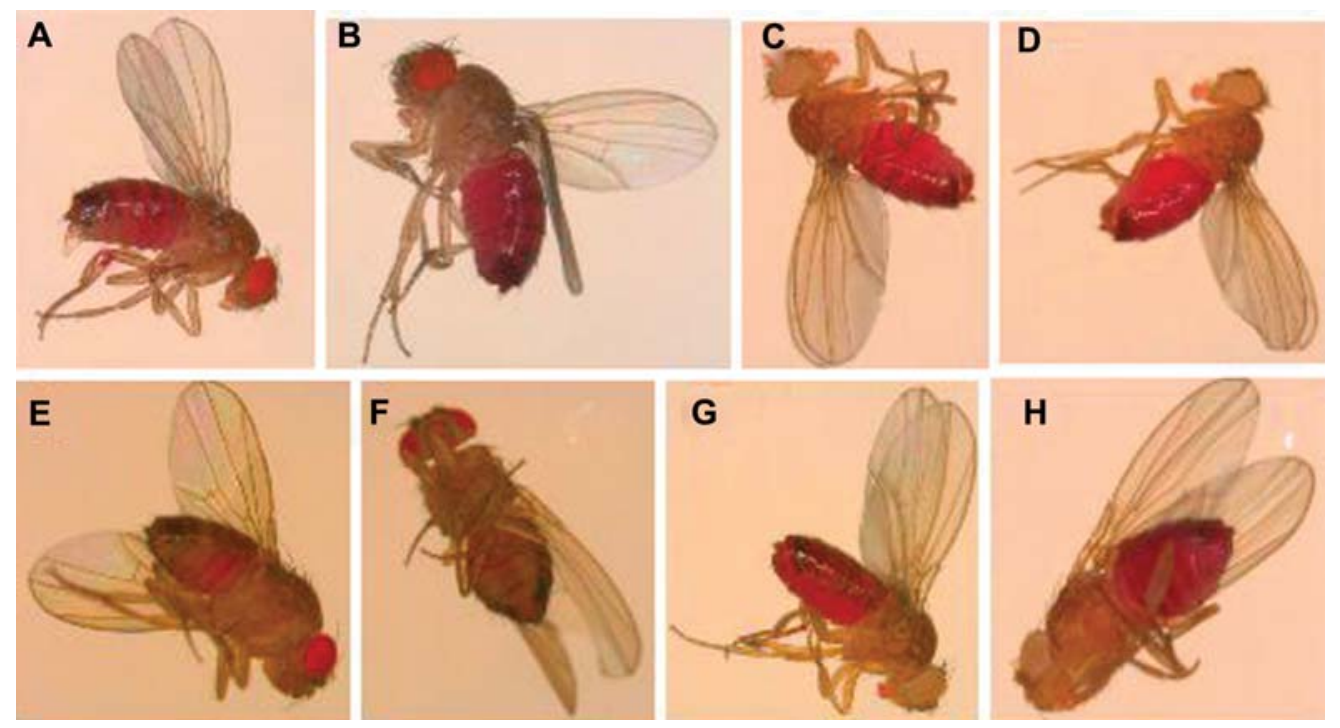

Figure 2 Gustatory assay with adult flies on normal or zinc-supplemented food with acid red dye as a marker.

(A, B) $\operatorname{park}^{25 /+}$ control flies and (C, D) $\operatorname{park}^{25 / 25}$ flies show the same feeding behavior on normal food, exemplified by the fully red abdomens. A food supplement of $4 \mathrm{~mm} \mathrm{Zn} \mathrm{(E,} \mathrm{F)} \mathrm{altered} \mathrm{feeding} \mathrm{behavior} \mathrm{of} \mathrm{park}{ }^{25 /+}$ (only partially red abdomens) but no change in the behavior of park $^{25 / 25}$ flies was observed $(\mathrm{G}, \mathrm{H})$ (fully red abdomens). The mutants are recognized by white eyes because of the loss of $\mathrm{w}^{+}$ (red eyes) which are present in control flies.

Methods: the adult gustatory assay was essentially carried out as described by A. Hilliker and colleagues (Bahadorani, 2008). Briefly, newly eclosed flies were reared on normal food for 2-3 days, then starved for $18 \mathrm{~h}$ on Whatman paper soaked with distilled water. After this treatment, starved flies (20 per vial) were transferred onto zinc-supplemented food with $0.2 \%$ sulforhodamine B sodium salt (acid red) for $2 \mathrm{~h}$. For control flies, culture medium was supplemented with $0.2 \%$ acid red, without metal supplement. After $2 \mathrm{~h}$ of feeding at optimum temperature $\left(25^{\circ} \mathrm{C}\right)$ and relative humidity, flies were anesthetized and the degree of abdomen redness was visually inspected. Abdomen redness was used as an indicator of the amount of food taken up.

moters by zinc and other metals in a cell-free transcription system (Zhang et al., 2003).

Zinc is not redox-active, but nevertheless toxic when in excess (Beyersmann and Haase, 2001). Acute zinc toxicity is rare but has been reported (Duncan et al., 1992; Whittaker, 1998; Prasad et al., 1999). If the extracellular concentration of zinc exceeds the capacity of zinc homeostasis mechanisms, it becomes cytotoxic and an excess of free intracellular zinc can trigger apoptosis (Choi et al., 1988; Duncan et al., 1992; Kim et al., 1999; Beyersmann and Haase, 2001; Wilhelm et al., 2001; Beyersmann, 2002; Walther et al., 2003). Zinc transport in Drosophila, as in vertebrates, is mediated by two families of solute linked carrier proteins: zinc importers (ZIPs), which function in the uptake of zinc to the cytoplasm, and zinc exporters (ZnTs), which reduce cytoplasmic zinc concentrations by promoting zinc efflux (Liuzzi and Cousins, 2004; Yepiskoposyan et al., 2006). More than 10 zinc transporter genes are annotated in Drosophila melanogaster based on their sequence similarities to vertebrate zinc transporters. The ZIP family gene foi (fear of intimacy) was characterized in Drosophila and shown to be a zinc importer that is critical during development (Moore et al., 1998; Mathews et al., 2005). Transcriptional responses to zinc in Drosophila larvae were analyzed in our laboratory (Yepiskoposyan et al., 2006). Apart from the expected upregulation of metallothioneins and the zinc exporters ZnT35C and $\mathrm{ZnT63C}$, there was also an induction of neurotransmitters, detoxification enzymes (such as glutathione S-transferase), ferritin and chaperone encoding genes.

We found that whereas parkin mutant flies readily feed on high-zinc food, their wild type counterparts avoid zinc-loaded food. The mutants also had an increased survival rate on zinc-supplemented food, which prompted us to investigate their response to zinc in more detail.

With standard 'normal food' (NF), parkin mutant flies have a median lifespan of 6 days with a maximum of 11 days (Figure 1A). Zinc supplementation in the form of zinc chloride increased the lifespan of parkin mutants. When maintained on supplements of $4 \mathrm{~mm} \mathrm{Zn}$, the mutant flies survived up to 23 days with a median lifespan of 8 days (Figure 1). This increase was as a result of zinc and not chloride ions, as similar survival assays on $\mathrm{NaCl}$-supplemented medium did not extend lifespan (data not shown). Zinc supplementation also increases the eclosion frequency of parkin mutants, from $2.5 \%$ to $19 \%$, i.e., close to $25 \%$ which is the expected frequency of parkin mutants in the progeny of parkin heterozygous parents (Table 1). In contrast, heterozygous control flies did not draw any benefit from zinc-supplemented food: if kept on food with $4 \mathrm{~mm} \mathrm{ZnCl}_{2}$, the median lifespan reduced significantly to 17 days, whereas on NF, $80 \%$ were still alive at the end of the experiment ( 23 days) (Figure 1A). Similar adverse effects of zinc load were 
observed with wild type $y w$ and OregonR flies (data not shown). To determine if zinc has a stronger effect during development from eggs to adults or during the adult feeding stage, we followed the lifespan of both parkin mutant and parkin heterozygous flies under four conditions: (i) development and adult maintenance in zinc supplement, (ii) raising the flies on NF until adult stage and then maintaining them on zinc-supplemented food post-eclosion, (iii) raising the flies on zinc food but maintaining them on NF after eclosion, and (iv) raising and maintaining the flies on NF. We observed that the strongest positive effect of zinc on park $^{25 / 25}$ flies was when they were both raised and maintained on $4 \mathrm{~mm}$ Zn-supplemented food (Figure 1B). The strongest negative effect of zinc on park $^{25 /+}$ control flies was also observed under these conditions (Figure 1C). Development of park $^{25 /+}$ and OregonR wild type flies was delayed but generally less affected by zinc than the survival of adults; egg transfer (200 each) from NF to vials containing increasing concentrations of zinc resulted in an equal percentage of eclosing adults in NF, 4 and $6 \mathrm{~mm} \mathrm{Zn}$ but none developed in $10 \mathrm{~mm} \mathrm{Zn} \mathrm{(data} \mathrm{not} \mathrm{shown;} \mathrm{see} \mathrm{also} \mathrm{Egli} \mathrm{et} \mathrm{al.,} \mathrm{2003).}$

parkin mutants might sense a deficiency in and/or a rescuing effect of zinc, and in response to this eat normal amounts of zinc-supplemented food unlike heterozygous or wild type flies. This was indicated by a visual inspection of adult Drosophila in a gustatory test and was quantitatively confirmed by the measurement of total zinc uptake by flies (Figures 2 and 3). On NF, the zinc content in heads of parkin mutant flies was much lower than in controls, consistent with a zinc deficiency in the mutants. Zinc supplementation indeed resulted in an, albeit minor, increase of zinc content in mutant heads (Figure 3A), whereas in thoraces the zinc content was similar in mutant and control flies (Figure 3B). Interestingly, the abdomens of park $^{25 / 25}$ flies fed on zinc food showed a 10-fold increase in zinc levels compared with flies fed on NF (Figure 3C). Such an effect was, however, not observed in the control flies which only showed a minor increase in zinc levels upon zinc feeding, which is in line with their tendency to avoid zinc-loaded food.

We also determined the transcript levels of parkin, MTF1 and some other genes involved in zinc import/export (Figure 4). As expected, parkin mutants have no detectable parkin transcripts. In comparison to control flies, transcript levels of the metallothionein $M t n B$ are higher in parkin mutants (Figure 4A,B). Zinc supplementation induced MtnB transcripts 24-fold in parkin mutants; in control flies the increase was 14-fold (Figure 4B). Boosting expression of metallothioneins, which act as ROS scavengers, is one way zinc could play an antioxidant role. The elevated metallothionein levels could also explain at least in part why an increased zinc concentration is not toxic to the parkin mutants. We also determined the transcript levels of three zinc transporters, the exporters ZnT35C and ZnT63C and the importer foi (Mathews et al., 2005; Yepiskoposyan et al., 2006) both in parkin mutants and in the heterozygous controls. The most conspicuous difference was observed with the exporter gene ZnT35C, a known target of MTF-1. In parkin mutants, expression was dramatically reduced com-
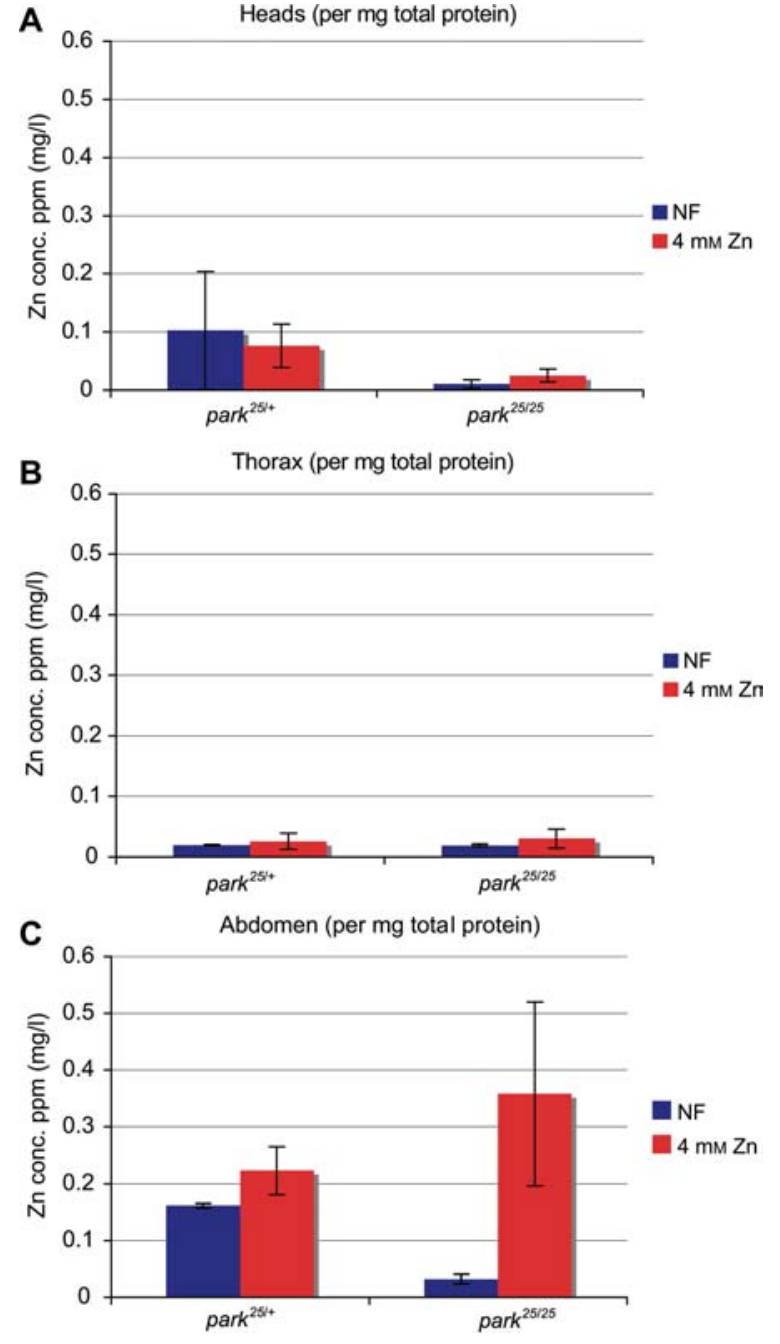

Figure 3 Quantification of $\mathrm{Zn}$ content in the different body parts of park $^{25 /+}$ control flies and park $^{25 / 25}$ flies.

Flies were raised on normal food (NF) or zinc-supplemented food (4 mm Zn). (A) Heads, (B) thoraces, (C) abdomens.

Methods: female flies were allowed to lay eggs on zinc-supplemented $\left(4 \mathrm{mM} \mathrm{ZnCl}_{2}\right)$ fly food for 6 days and removed afterwards. The resulting progeny was collected at regular intervals and the percentage of eclosing parkin homozygous mutants and heterozygous controls were calculated each time. These progeny were then frozen and stored until the required number of 200 flies was obtained in triplicate for each genotype. Frozen flies were then dissected to separate their heads, thoraces and abdomens which were collected separately. Each sample set was then subjected to homogenization using cold protein homogenization buffer $(0.32 \mathrm{~mm}$ sucrose, $20 \mathrm{~mm}$ HEPES, $1 \mathrm{~mm} \mathrm{MgCl}_{2}, 0.5 \mathrm{~mm}$ PMSF protease inhibitor at $\mathrm{pH}$ 7.4) and the samples were normalized for protein content. A highly sensitivity flame atomic absorption spectrophotometer (FAAS; GTA-120/PSD-120, Varian Australia Pty Ltd, Mulgrave, VIC, Australia) was used to detect the zinc content in each body part of each genotype assessed.

pared to controls and not responsive to zinc. In heterozygous controls, zinc supplement resulted in a 1.5-fold upregulation, which indicates a response to zinc overload (Figure 4C). An increased expression of zinc exporters in control flies is in 


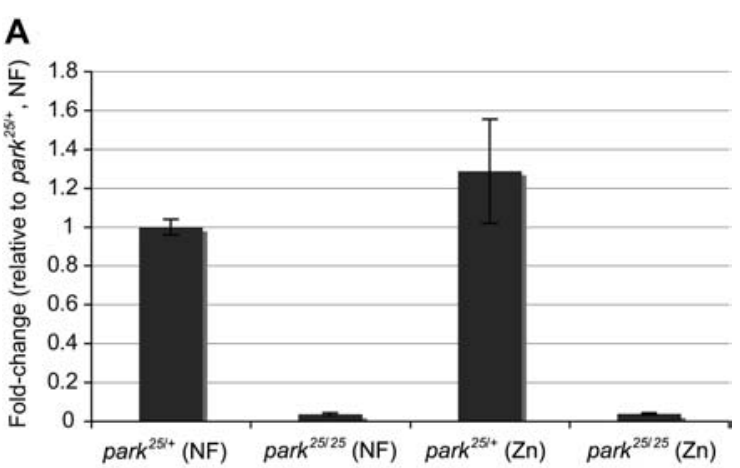

B

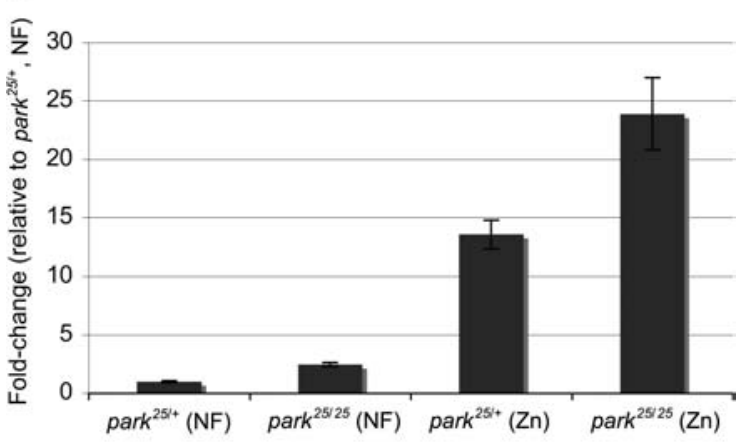

C

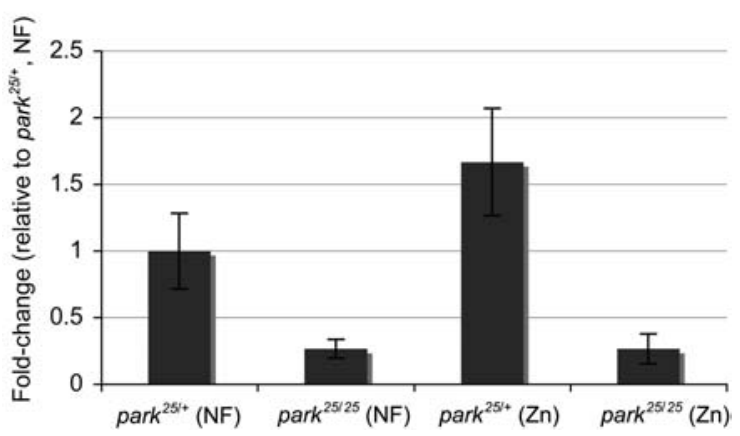

agreement with their avoidance of zinc-supplemented food. Transcripts of ZnT63C and, somewhat unexpectedly of foi, were also lower in parkin mutants compared to parkin heterozygous controls (data not shown). Taken together, these data suggest that zinc homeostasis is distorted in parkin mutants, with the effect that zinc supplement in food produces a strong phenotypic rescue effect. It remains to be seen whether zinc supplementation also has a positive effect in a mouse model of familial PD and ultimately in Parkinson's patients.

\section{Acknowledgments}

We thank Drs. Jessica Greene and Leo Pallanck (University of Washington) for providing the $\operatorname{park}^{25 / 25}$ flies, Drs. Eva Freisinger and Augusto Cabral for the metal quantification experiments, Martin Moser for the RT-PCR analysis, Ivan Ostojic (Friedrich Miescher Institute, Basel, Switzerland) for statistical analyses, Till Strassen for maintenance of fly stocks and Dr. George Hausmann for critical
Figure 4 Real-time analysis of parkin transcript levels. (A) In park $^{25 / 25}$ flies no parkin transcripts are detectable. In park ${ }^{25 /+}$ parkin transcripts are, if anything, elevated in flies raised on zinc food. (B) park $^{25 / 25}$ flies have increased levels of metallothionein $M t n B$ transcripts in NF compared to control flies. Zinc induces $M t n B$ in both genotypes, but to a higher level in $\operatorname{park}^{25 / 25}$. (C) The zinc exporter, ZnT35C, shows increased transcript levels in park $^{25 /+}$ on zinc food, which is not observed in park $^{25 / 25}$ flies.

Methods: total RNA was purified from the adult Drosophila tissue using the nucleospin RNA II protocol and eluted in $60 \mu 1$ RNasefree water. cDNA was prepared using the transcriptor high fidelity cDNA synthesis kit from Roche (Mannheim, Germany). The cDNA obtained was further purified using the AM 1906 DNA-free kit (Ambion, Rotkreuz, Switzerland). This was then used for analysis by real-time PCR on the Tecan Genesis 200/8 robot (Tecan, Männedorf, Switzerland) using the Mesa Green qPCR Mastermix Plus (Eurogentec, Seraing, Belgium) for SYBR assays. The qPCR run was performed on an Applied Biosystems machine (ABI Prism SDS 7900 HAT; Seraing, Belgium) in a 384-well format with a reaction volume of $10 \mu \mathrm{l}$. All fold-change values are normalized to corresponding park $^{25 /+}$ values on normal food (NF). Housekeeping genes used for reference were actin $5 C$, sec 24 and GAPDH. The primer sequences used for the transcripts quantified were: for parkin, $5^{\prime}$ AAG ATC ATA TTT GCC GGT AAG GAA-3' and 5'-CGC TTT GCT GAC CCA AGT C-3' amplify a 73-bp fragment only from the parkin heterozygous control flies; for MtnB, 5'-TTG CAA GGG TTG TGG AAC AAA- $3^{\prime}$ and $5^{\prime}$-TGC AGG CGC AGT TGT CC$3^{\prime}$ amplify a 65-bp fragment, and for ZnT35C, 5'-GCT CTC GCC GAT CTG AAA GT-3' and 5'-CGC ACC GAC AAG TGT TTC TTA TA-3' amplify a 75-bp fragment.

reading of the manuscript. This work was supported by the Kanton Zürich and the Swiss National Science Foundation.

\section{References}

Andrews, G.K. (2000). Regulation of metallothionein gene expression by oxidative stress and metal ions. Biochem. Pharmacol. 59, 95-104.

Berg, J.M. and Shi, Y. (1996). The galvanization of biology: a growing appreciation for the roles of zinc. Science 271, 1081-1085.

Beyersmann, D. (2002). Effects of carcinogenic metals on gene expression. Toxicol. Lett. 127, 63-68.

Beyersmann, D. and Haase, H. (2001). Functions of zinc in signaling, proliferation and differentiation of mammalian cells. Biometals $14,331-341$.

Bray, T.M. and Bettger, W.J. (1990). The physiological role of zinc as an antioxidant. Free Radic. Biol. Med. 8, 281-291.

Bueler, H. (2009). Impaired mitochondrial dynamics and function in the pathogenesis of Parkinson's disease. Exp. Neurol. 218, 235-246.

Choi, D.W., Yokoyama, M., and Koh, J. (1988). Zinc neurotoxicity in cortical cell culture. Neuroscience 24, 67-79.

Choi, H.Y., Song, J.H., Park, D.K., and Ross, G.M. (2000). The effects of ascorbic acid on dopamine-induced death of PC12 cells are dependent on exposure kinetics. Neurosci. Lett. 296, $81-84$.

Dawson, T.M. and Dawson, V.L. (2003). Molecular pathways of neurodegeneration in Parkinson's disease. Science 302, 819822 . 
Dexter, D.T., Carter, C.J., Wells, F.R., Javoy-Agid, F., Agid, Y., Lees, A., Jenner, P., and Marsden, C.D. (1989). Basal lipid peroxidation in substantia nigra is increased in Parkinson's disease. J. Neurochem. 52, 381-389.

Duncan, M.W., Marini, A.M., Watters, R., Kopin, I.J., and Markey, S.P. (1992). Zinc, a neurotoxin to cultured neurons, contaminates cycad flour prepared by traditional guamanian methods. J. Neurosci. 12, 1523-1537.

Egli, D., Selvaraj, A., Yepiskoposyan, H., Zhang, B., Hafen, E., Georgiev, O., and Schaffner, W. (2003). Knockout of 'metalresponsive transcription factor' MTF-1 in Drosophila by homologous recombination reveals its central role in heavy metal homeostasis. EMBO J. 22, 100-108.

Gasser, T. (2005). Genetics of Parkinson's disease. Curr. Opin. Neurol. $18,363-369$.

Goldberg, M.S., Fleming, S.M., Palacino, J.J., Cepeda, C., Lam, H.A., Bhatnagar, A., Meloni, E.G., Wu, N., Ackerson, L.C., Klapstein, G.J., et al. (2003). Parkin-deficient mice exhibit nigrostriatal deficits but not loss of dopaminergic neurons. J. Biol. Chem. 278, 43628-43635.

Greene, J.C., Whitworth, A.J., Kuo, I., Andrews, L.A., Feany, M.B., and Pallanck, L.J. (2003). Mitochondrial pathology and apoptotic muscle degeneration in Drosophila parkin mutants. Proc. Natl. Acad. Sci. USA 100, 4078-4083.

Greene, J.C., Whitworth, A.J., Andrews, L.A., Parker, T.J., and Pallanck, L.J. (2005). Genetic and genomic studies of Drosophila parkin mutants implicate oxidative stress and innate immune responses in pathogenesis. Hum. Mol. Genet. 14, 799-811.

Itier, J.M., Ibanez, P., Mena, M.A., Abbas, N., Cohen-Salmon, C., Bohme, G.A., Laville, M., Pratt, J., Corti, O., Pradier, L., et al. (2003). Parkin gene inactivation alters behaviour and dopamine neurotransmission in the mouse. Hum. Mol. Genet. 12, 22772291.

Jenner, P. and Olanow, C.W. (1996). Oxidative stress and the pathogenesis of Parkinson's disease. Neurology 47, S161-S170.

Kim, E.Y., Koh, J.Y., Kim, Y.H., Sohn, S., Joe, E., and Gwag, B.J. (1999). $\mathrm{Zn}^{2+}$ entry produces oxidative neuronal necrosis in cortical cell cultures. Eur. J. Neurosci. 11, 327-334.

Kocaturk, S., Kocaturk, P.A., Kavas, G.O., and Mutluer, N. (1996). Antioxidant defence system in a patient with cerebrovascular accident. J. Int. Med. Res. 24, 376-380.

Lichtlen, P. and Schaffner, W. (2001). Putting its fingers on stressful situations: the heavy metal-regulatory transcription factor MTF1. Bioessays 23, 1010-1017.

Liuzzi, J.P. and Cousins, R.J. (2004). Mammalian zinc transporters. Annu. Rev. Nutr. 24, 151-172.

Mathews, W.R., Wang, F., Eide, D.J., and Van Doren, M. (2005). Drosophila fear of intimacy encodes a Zrt/IRT-like protein (ZIP) family zinc transporter functionally related to mammalian ZIP proteins. J. Biol. Chem. 280, 787-795.

Moore, L.A., Broihier, H.T., Van Doren, M., and Lehmann, R. (1998). Gonadal mesoderm and fat body initially follow a common developmental path in Drosophila. Development 125, 837-844.

Olanow, C.W. and Tatton, W.G. (1999). Etiology and pathogenesis of Parkinson's disease. Annu. Rev. Neurosci. 22, 123-144.

Palacino, J.J., Sagi, D., Goldberg, M.S., Krauss, S., Motz, C., Wacker, M., Klose, J., and Shen, J. (2004). Mitochondrial dysfunction and oxidative damage in parkin-deficient mice. J. Biol. Chem. 279, 18614-18622.

Parkin, G. (2004). Synthetic analogues relevant to the structure and function of zinc enzymes. Chem. Rev. 104, 699-767.
Pesah, Y., Pham, T., Burgess, H., Middlebrooks, B., Verstreken, P., Zhou, Y., Harding, M., Bellen, H., and Mardon, G. (2004). Drosophila parkin mutants have decreased mass and cell size and increased sensitivity to oxygen radical stress. Development 131 , 2183-2194.

Prasad, A.S., Beck, F.W., Kaplan, J., Chandrasekar, P.H., Ortega, J., Fitzgerald, J.T., and Swerdlow, P. (1999). Effect of zinc supplementation on incidence of infections and hospital admissions in sickle cell disease (SCD). Am. J. Hematol. 61, 194-202.

Riparbelli, M.G. and Callaini, G. (2007). The Drosophila parkin homologue is required for normal mitochondrial dynamics during spermiogenesis. Dev. Biol. 303, 108-120.

Sang, T.K., Chang, H.Y., Lawless, G.M., Ratnaparkhi, A., Mee, L., Ackerson, L.C., Maidment, N.T., Krantz, D.E., and Jackson, G.R. (2007). A Drosophila model of mutant human parkininduced toxicity demonstrates selective loss of dopaminergic neurons and dependence on cellular dopamine. J. Neurosci. 27, 981-992.

Saydam, N., Georgiev, O., Nakano, M.Y., Greber, U.F., and Schaffner, W. (2001). Nucleo-cytoplasmic trafficking of metal-regulatory transcription factor 1 is regulated by diverse stress signals. J. Biol. Chem. 276, 25487-25495.

Smirnova, I.V., Bittel, D.C., Ravindra, R., Jiang, H., and Andrews, G.K. (2000). Zinc and cadmium can promote rapid nuclear translocation of metal response element-binding transcription factor1. J. Biol. Chem. 275, 9377-9384.

Suzuki, T., Umeyama, T., Ohma, C., Yamanaka, H., Suzuki, K., Nakajima, K., and Kimura, M. (1991). Immunohistochemical study of metallothionein in normal and benign prostatic hyperplasia of human prostate. Prostate 19, 35-42.

Valko, M., Morris, H., and Cronin, M.T. (2005). Metals, toxicity and oxidative stress. Curr. Med. Chem. 12, 1161-1208.

Walther, U.I., Czermak, A., Muckter, H., Walther, S.C., and Fichtl, B. (2003). Decreased GSSG reductase activity enhances cellular zinc toxicity in three human lung cell lines. Arch. Toxicol. 77, $131-137$.

Warner, T.T. and Schapira, A.H. (2003). Genetic and environmental factors in the cause of Parkinson's disease. Ann. Neurol. 53 (Suppl. 3), S16-S23; discussion S23-S25.

West, A.B. and Maidment, N.T. (2004). Genetics of parkin-linked disease. Hum. Genet. 114, 327-336.

Whittaker, P. (1998). Iron and zinc interactions in humans. Am. J. Clin. Nutr. 68, 442S-446S.

Whitworth, A.J., Theodore, D.A., Greene, J.C., Benes, H., Wes, P.D., and Pallanck, L.J. (2005). Increased glutathione S-transferase activity rescues dopaminergic neuron loss in a Drosophila model of Parkinson's disease. Proc. Natl. Acad. Sci. USA 102, 8024-8029.

Wilhelm, B., Walther, U.I., and Fichtl, B. (2001). Effects of zinc chloride on glutathione and glutathione synthesis rates in various lung cell lines. Arch. Toxicol. 75, 388-394.

Yepiskoposyan, H., Egli, D., Fergestad, T., Selvaraj, A., Treiber, C., Multhaup, G., Georgiev, O., and Schaffner, W. (2006). Transcriptome response to heavy metal stress in Drosophila reveals a new zinc transporter that confers resistance to zinc. Nucleic Acids Res. 34, 4866-4877.

Zhang, B., Georgiev, O., Hagmann, M., Gunes, C., Cramer, M., Faller, P., Vasak, M., and Schaffner, W. (2003). Activity of metal-responsive transcription factor 1 by toxic heavy metals and $\mathrm{H}_{2} \mathrm{O}_{2}$ in vitro is modulated by metallothionein. Mol. Cell. Biol. $23,8471-8485$.

Received January 15, 2010; accepted January 26, 2010 